Available on line at www.rac.es/racsam Applied Mathematics
REVISTA DE LA REAL ACADEMIA DE CIENCIAS EXACTAS, FISICAS Y NATURALES. $\mathbb{S E R} \mathbb{E} \mathbb{A}: \mathbb{M} \mathbb{A} \mathbb{E M} \mathbb{A} \mathbb{T} \mathbb{C A S}$ Madrid (España / Spain)

\title{
Nonlinear evolution equations on locally closed graphs
}

\author{
Mihai Necula, Marius Popescu and Ioan I. Vrabie
}

\begin{abstract}
Let $X$ be a real Banach space, let $A: \underline{D(A)} \subseteq X \rightsquigarrow X$ be an $m$-dissipative operator, let $I$ a nonempty, bounded interval and let $K: I \rightsquigarrow \overline{D(A)}$ be a given multi-valued function. By using the concept of A-quasi-tangent set introduced by Cârjă, Necula, Vrabie [8] and [9] and using a tangency condition expressed in the terms of this concept, we establish a necessary and sufficient condition for $C^{0}$-viability referring to nonlinear evolution inclusions of the form $u^{\prime}(t) \in A u(t)+F(t, u(t))$, where $F$ is a multi-function defined on the graph of $K$. As an application, we deduce a comparison result for a class of fully nonlinear evolution inclusions driven by multi-valued perturbations of subdifferentials.
\end{abstract}

\section{Ecuaciones de evolución no lineales en grafos localmente cerrados.}

Resumen. Sea $X$ un espacio de Banach real, sea $A: D(A) \subseteq X \rightsquigarrow X$ un operador $m$-disipativo, sea $I$ un intervalo acotado no vacío y sea $K: I \rightsquigarrow \overline{D(A)}$ una función multivaluada. Utilizando el concepto de conjunto $A$-casi-tangente introducido por Cârjă, Necula, Vrabie [8] y [9] y utilizando condiciones de tangencia expresadas en términos de este concepto, establecemos una condición necesaria y suficiente de $C^{0}$-viabilidad para inclusiones de evolución no lineales de la forma $u^{\prime}(t) \in A u(t)+F(t, u(t))$, donde $F$ es una multi-función definida en el grafo de $K$. Como aplicación, se deduce un resultado de comparación para una clase de inclusiones de evolución no lineales completas asociadas a perturbaciones multi-valuadas de subdiferenciales.

\section{Introduction}

Let $X$ be a real Banach space and let $A: D(A) \subseteq X \rightsquigarrow X$ be an $m$-dissipative operator, which means that $-A$ is $m$-accretive, generating the nonlinear semigroup of contractions $\{S(t): \overline{D(A)} \rightarrow \overline{D(A)} ; t \geq 0\}$. Let $K: I \rightsquigarrow \overline{D(A)}$ and $F: \mathcal{K} \rightsquigarrow X$ be two multi-functions with nonempty values, where $I \subseteq \mathbb{R}$ is a nonempty and open from the right interval and $\mathcal{K}:=\operatorname{graph}(K)$.

Submitted by Ildefonso Díaz

Received: March 20, 2009. Accepted: April 1, 2009

Keywords: Differential inclusion, locally closed graph, tangent set, tangency condition, multi-valued mapping, viability

Mathematics Subject Classifications: Primary 34G25; Secondary 34A60, 47H20, 34C11

(c) 2010 Real Academia de Ciencias, España 
Our aim here is to prove some new necessary and sufficient conditions in order that $\mathcal{K}$ be viable with respect to $A+F$. To be more precise, let us consider the Cauchy Problem

$$
\left\{\begin{array}{l}
u^{\prime}(t) \in A u(t)+F(t, u(t)) \\
u(\tau)=\xi
\end{array}\right.
$$

The next concept is widely known, after the pioneering work of Crandall, Liggett [12], under the name of mild solution and, within the frame here considered, coincides with the one of integral solution introduced by Benilan [2].

Definition 1 Let $A: D(A) \subseteq X \rightsquigarrow X$ be $m$-dissipative and let $f \in L^{1}(\tau, T ; X)$. $A C^{0}$-solution, or $D S$-limit solution, of the equation

$$
u^{\prime}(t) \in A u(t)+f(t)
$$

is a function $u$ in $C([\tau, T] ; X)$ satisfying: for each $\tau<c<T$ and $\varepsilon>0$ there exist

(i) $\tau=t_{0}<t_{1}<\cdots<c \leq t_{n}<T, \quad t_{k}-t_{k-1} \leq \varepsilon \quad$ for $k=1,2, \ldots, n$;

(ii) $f_{1}, \ldots, f_{n} \in X \quad$ with $\sum_{k=1}^{n} \int_{t_{k-1}}^{t_{k}}\left\|f(t)-f_{k}\right\| \mathrm{d} t \leq \varepsilon$;

(iii) $v_{0}, \ldots, v_{n} \in X \quad$ satisfying:

$$
\begin{aligned}
& \frac{v_{k}-v_{k-1}}{t_{k}-t_{k-1}} \in A v_{k}+f_{k} \quad \text { for } k=1,2, \ldots, n \quad \text { and such that } \\
& \left\|u(t)-v_{k}\right\| \leq \varepsilon \quad \text { for } t \in\left[t_{k-1}, t_{k}\right), k=1,2, \ldots, n .
\end{aligned}
$$

Definition 2 A function $u:[\tau, T] \rightarrow X$ is a $C^{0}$-solution of (1) if $u(\tau)=\xi, u(t) \in K(t)$ for each $t \in[\tau, T]$, and there exists an a.e. selection $f \in L^{1}(\tau, T ; X)$ of $t \mapsto F(t, u(t))$, i.e., $f(t) \in F(t, u(t))$ a.e. for $t \in[\tau, T]$, such that $u$ is a $C^{0}$-solution on $[\tau, T]$ of the equation (2) in the usual sense.

The next two classical results will prove useful in that follows.

Theorem 1 Let $X$ be a Banach space and let $A: D(A) \subseteq X \rightsquigarrow X$ be $m$-dissipative. Then, for each $\xi \in \overline{D(A)}$ and $f \in L^{1}(\tau, T ; X)$, there exists a unique $C^{0}$-solution $u:[\tau, T] \rightarrow \overline{D(A)}$, of (2), which satisfies $u(\tau)=\xi$.

Proof. See Lakshmikantham-Leela [14, Theorem 3.6.1, p. 116].

In order to exhibit the dependence of the $C^{0}$-solution $u$ of (2) on $\tau, \xi$ and $f$, we denote it by $u=$ $u(\cdot, \tau, \xi, f)$. Throughout, $[x, y]_{+}$denotes the right directional derivative of the norm calculated at $x$ in the direction $y$, i.e.,

$$
[x, y]_{+}=\lim _{h \downarrow 0} \frac{1}{h}(\|x+h y\|-\|x\|) .
$$

Similarly,

$$
(x, y)_{+}=\lim _{h \downarrow 0} \frac{1}{2 h}\left(\|x+h y\|^{2}-\|x\|^{2}\right) .
$$

Theorem 2 Let $X$ be a Banach space, let $A: D(A) \subseteq X \rightsquigarrow X$ be $m$-dissipative, let $\xi, \eta \in \overline{D(A)}, f$, $g \in L^{1}(\tau, T ; X)$ and let $\widetilde{u}=u(\cdot, \tau, \xi, f)$ and $\widetilde{v}=u(\cdot, \tau, \eta, g)$. We have

$$
\|\widetilde{u}(t)-\widetilde{v}(t)\| \leq\|\xi-\eta\|+\int_{\tau}^{t}[\widetilde{u}(s)-\widetilde{v}(s), f(s)-g(s)]_{+} \mathrm{d} s
$$


and

$$
\|\widetilde{u}(t)-\widetilde{v}(t)\|^{2} \leq\|\xi-\eta\|^{2}+2 \int_{\tau}^{t}(\widetilde{u}(s)-\widetilde{v}(s), f(s)-g(s))_{+} \mathrm{d} s,
$$

for each $t \in[\tau, T]$. Moreover, we have the following evolution property

$$
u(t, a, \xi, f)=u\left(t, \nu, u(\nu, a, \xi, f),\left.f\right|_{[\nu, \nu+\delta]}\right),
$$

for $\tau \leq a \leq \nu \leq t \leq \nu+\delta$

Proof. See Vrabie [25, Section 1.7].

Since for each $x, y \in X,[x, y]_{+} \leq\|y\|$, from Theorem 2 , we deduce

Corollary 1 Let $X$ be a Banach space, let $A: D(A) \subseteq X \rightsquigarrow X$ be m-dissipative, let $\xi, \eta \in \overline{D(A)}$, $f, g \in L^{1}(\tau, T ; X)$ and let $\widetilde{u}=u(\cdot, \tau, \xi, f)$ and $\widetilde{v}=u(\cdot, \tau, \eta, g)$. We have

$$
\|\widetilde{u}(t)-\widetilde{v}(t)\| \leq\|\widetilde{u}(s)-\widetilde{v}(s)\|+\int_{s}^{t}\|f(\theta)-g(\theta)\| \mathrm{d} \theta
$$

for each $\tau \leq s \leq t \leq T$.

Definition 3 We say that the graph, $\mathcal{K}$, of $K: I \rightsquigarrow \overline{D(A)}$, is $C^{0}$-viable with respect to $A+F$, where $F: \mathcal{K} \rightsquigarrow X$, if for each $(\tau, \xi) \in \mathcal{K}$, there exists $T>\tau$, such that $[\tau, T] \subseteq I$ and (1) has at least one $C^{0}$-solution $u:[\tau, T] \rightarrow X$. If $T \in(\tau, \sup I)$ can be taken arbitrary, we say that $\mathcal{K}$ is globally $C^{0}$-viable with respect to $A+F$.

A short review of the main contributions to the viability theory for evolution inclusions is given below. Roughly speaking, the $S$-viability of a set, $K$, with respect to the right-hand side of an evolution inclusion means that for each $\xi \in K$ there exists at least one $S$-solution $u$ of the evolution inclusion in question satisfying $u(\tau)=\xi$ and $u(t) \in K$ for each $t \in[\tau, T]$. Here $S$ means the sense in which the term solution should be understood, sense which has to be made very precise case by case.

Throughout, if $u \in X, B \subseteq X$ and $C \subseteq X$, we denote by

$$
\operatorname{dist}(u, C)=\inf _{v \in C}\|u-v\|, \quad \operatorname{dist}(B, C)=\inf _{\substack{v \in B \\ w \in C}}\|v-w\| \quad \text { and } \quad\|B\|=\sup _{v \in B}\|v\|
$$

Emerged from its classical ordinary differential equations counterpart initiated by Perron [22] in the one-dimensional case and extended by Nagumo [16] to the arbitrary but finite dimensions, the viability theory for ordinary differential inclusions of the form $u^{\prime}(t) \in F(u(t))$ started with the paper of Bebernes, Shuur [1] where they have shown that, whenever $F: K \rightsquigarrow X$ is upper semi-continuous (u.s.c.) with nonempty, convex, closed and bounded values, and $K \subseteq X$ is locally closed, a necessary and sufficient condition in order that $K$ be absolutely continuous-viable with respect to $F$ is

$$
F(\xi) \cap \mathcal{T}_{K}(\xi) \neq \emptyset
$$

for each $\xi \in K$, where $\mathcal{T}_{K}(\xi)$ denotes the contingent cone to $K$ at $\xi \in K$. We recall that $\mathcal{T}_{K}(\xi)$ consists of all vectors $\eta \in X$ which are tangent to $K$ at $\xi \in K$ in the sense of Bouligand [5] and Severi [23], i.e.,

$$
\liminf _{h \downarrow 0} \frac{1}{h} \operatorname{dist}(\xi+h \eta ; K)=0 .
$$

Clearly, (4) is nothing but a simple extension of the well-known tangency condition: $f(\xi) \in \mathcal{T}_{K}(\xi)$ for each $\xi \in K$, used by Nagumo [16] in the single-valued and autonomous case, i.e. $F(\xi)=\{f(\xi)\}$. Extensions of the main result of Bebernes-Schuur [1], to multi-functions defined on graphs, in finite dimensional 
spaces, were obtained subsequently by Methlouthi [15], for u.s.c. $F$, and by Bothe [3], for almost u.s.c. $F$. For similar results in infinite dimensional spaces, see Bothe [4], as well as Cârjă, Necula, Vrabie [9].

Recently, Cârjă, Necula, Vrabie [10] considered the multi-valued perturbed case $u^{\prime}(t) \in A u(t)+F(u(t))$ with $A$ the infinitesimal generator of a $C_{0}$-semigroup and $F: K \rightsquigarrow X$. In order to cover more general situations, from the viewpoints of both necessary and sufficient conditions of mild-viability, they introduced the concept of A-quasi-tangent set to a given set $K$ at a given point $\xi \in K$ by saying that a nonempty and bounded subset $E$ in $X$ is $A$-quasi-tangent to $K$ at $\xi \in K$ if

$$
\liminf _{h \downarrow 0} \frac{1}{h} \operatorname{dist}\left(S(h) \xi+\int_{0}^{h} S(h-s) \mathcal{F}_{E} \mathrm{~d} s ; K\right)=0,
$$

where

$$
\mathcal{F}_{E}=\left\{f \in L_{\mathrm{loc}}^{1}(\mathbb{R} ; X) ; f(s) \in E \text { a. e. for } s \in \mathbb{R}\right\} .
$$

We notice that this concept is strictly more general than that one of $A$-tangent vector used by Pavel [20] in the single-valued case, and by Pavel, Vrabie [21] in the multi-valued case. Subsequently, inspired by the notion of $A$-tangent vector, with $A$ nonlinear, used by Vrabie [24], they have extended this concept to the case in which $A$ is nonlinear and have proved some necessary and sufficient conditions of $C^{0}$-viability. See Cârjă, Necula, Vrabie [8] and [11].

By imposing a tangency condition expressed in terms of $A$-quasi-tangent sets, in this paper, we extend the main result in Necula, Popescu, Vrabie [19] to the fully nonlinear case, by proving a sufficient, and even a necessary and sufficient condition for $C^{0}$-viability referring to nonlinear evolution inclusions driven by multi-valued nonautonomous and $t$-discontinuous perturbations defined on graphs. The advantage of using $A$-quasi-tangent sets instead of $A$-tangent vectors consists in that, in infinite dimensions, the "multi-valued tangency condition" turns out to be not only sufficient for $C^{0}$-viability, but necessary as well.

Finally, it should be noticed that there are two main difficulties to overcome in this context. The first one consists in finding a suitable definition of the approximate solutions, in the general case here considered, i.e., that one of a multi-function defined on a non-cylindric domain, multi-function which may fail to be u.s.c. with respect to the $t$-variable. The second main difficult point here is to construct a sequence of approximate solutions which, under some additional fairly natural assumptions, has at least one convergent subsequence.

The paper is divided into 7 sections, the second and the third ones being merely concerned with the definition of both tangency concepts and special classes of multi-functions to be used in the sequel. The fourth section contains the main necessary condition of $C^{0}$-viability, while in the fifth section we state the main mild-viability sufficient condition and prove the existence of approximation solutions. In section 6 we prove the main sufficient condition for mild-viability, while the last section 7, as an application, we include a comparison result referring to a class of nonlinear evolution inclusions governed by multi-valued perturbations of subdifferentials.

\section{Tangency concepts}

Let $X$ be a real Banach space, $I \subseteq \mathbb{R}$ a nonempty and open from the right interval, let $K: I \rightsquigarrow \overline{D(A)}$ be a multi-function with nonempty values and let $\mathcal{K}$ be the graph of $K$, i.e. $\mathcal{K}:=\{(\tau, \xi) \in I \times X ; \tau \in I$, $\xi \in K(\tau)\}$. Let $(\tau, \xi) \in \mathcal{K}$, let $\eta \in X$ and let $E \in \mathcal{B}(X)$, where $\mathcal{B}(X)$ denotes the class of all nonempty and bounded subsets in $X$.

Definition 4 We say that

(i) the vector $\eta$ is $A$-tangent to $\mathcal{K}$ at $(\tau, \xi)$ if

$$
\liminf _{h \downarrow 0} \frac{1}{h} \operatorname{dist}(u(\tau+h, \tau, \xi, \eta) ; K(\tau+h))=0,
$$


where $u(\cdot, \tau, \xi, \eta)$ denotes the unique $C^{0}$-solution of the Cauchy problem

$$
\left\{\begin{array}{l}
v^{\prime}(t) \in A v(t)+\eta \\
v(\tau)=\xi
\end{array}\right.
$$

on $[\tau,+\infty)$. See Definition 1.

(ii) the set $E$ is $A$-tangent to $\mathcal{K}$ at $(\tau, \xi)$ if

$$
\liminf _{h \downarrow 0} \frac{1}{h} \operatorname{dist}(u(\tau+h, \tau, \xi, E) ; K(\tau+h))=0,
$$

where $u(\tau+h, \tau, \xi, E)=\{u(\tau+h, \tau, \xi, \eta) ; \eta \in E\}$.

(iii) $E$ is $A$-quasi-tangent to $\mathcal{K}$ at $(\tau, \xi)$ if

$$
\liminf _{h \downarrow 0} \frac{1}{h} \operatorname{dist}\left(u\left(\tau+h, \tau, \xi, \mathcal{F}_{E}\right) ; K(\tau+h)\right)=0,
$$

where $\mathcal{F}_{E}$ is defined by (5), and $u\left(\tau+h, \tau, \xi, \mathcal{F}_{E}\right)=\left\{u(\tau+h, \tau, \xi, f) ; f \in \mathcal{F}_{E}\right\}$.

Throughout, we denote by:

(i) $\mathcal{T}_{\mathcal{K}}^{A}(\tau, \xi)$ the set of all $A$-tangent vectors to $\mathcal{K}$ at $(\tau, \xi)$;

(ii) $\mathcal{T S}_{\mathcal{K}}^{A}(\tau, \xi)$ the set of all $A$-tangent sets to $\mathcal{K}$ at $(\tau, \xi)$;

(iii) $\mathcal{Q T S}_{\mathcal{K}}^{A}(\tau, \xi)$ the set of all $A$-quasi-tangent sets to $\mathcal{K}$ at $(\tau, \xi)$.

Identifying vectors with singletons, and constants with locally integrable functions, we deduce

$$
\mathcal{T}_{\mathcal{K}}^{A}(\tau, \xi) \subseteq \mathcal{T S}_{\mathcal{K}}^{A}(\tau, \xi) \subseteq Q \mathcal{T} \mathcal{S}_{\mathcal{K}}^{A}(\tau, \xi)
$$

and it may happen, even in the simplest case $A \equiv 0$, that both inclusions to be strict. See Example 2.4.1, p. 36 in Cârjă, Necula, Vrabie [9].

\section{Special classes of multi-functions}

Throughout, $\mathcal{K}$ is endowed with the metric, $d$, defined by

$$
d((\tau, \xi),(\theta, \mu))=\max \{|\tau-\theta|,\|\xi-\mu\|\}
$$

for all $(\tau, \xi),(\theta, \mu) \in \mathcal{K}$. Furthermore, whenever we will use the term strongly-weakly u.s.c. we will mean that the domain of the multi-function in question is equipped with the strong topology, while the range is equipped with the weak topology. The term u.s.c. refers to the case in which both domain and range are endowed with the strong, i.e. norm, topology. Finally, in all that follows, $\lambda$ stands for the Lebesgue measure.

Definition 5 The multi-function $F: \mathcal{K} \rightsquigarrow X$ is called (strongly-weakly) almost u.s.c. if for each $\varepsilon>0$ there exists an open set $\mathcal{O}_{\varepsilon} \subseteq I$ such that $\lambda\left(\mathcal{O}_{\varepsilon}\right) \leq \varepsilon$ and $F_{\mid\left[\left(I \backslash \mathcal{O}_{\varepsilon}\right) \times X\right] \cap \mathcal{K}}$ is (strongly-weakly) u.s.c.

Definition 6 We say that $F: \mathcal{K} \rightsquigarrow X$ is essentially locally bounded if, for each $(\tau, \xi) \in \mathcal{K}$, there exist a negligible set $N_{1} \subseteq I, \rho>0$, and $\ell_{1} \in L_{\mathrm{loc}}^{\infty}(I ; \mathbb{R})$ such that for all $\left.(t, u) \in\left(I \backslash N_{1}\right) \times D(\xi, \rho)\right) \cap \mathcal{K}$, we have

$$
\|F(t, u)\| \leq \ell_{1}(t)
$$

If we relax the condition $\ell_{1} \in L_{\mathrm{loc}}^{\infty}(I ; \mathbb{R})$ to $\ell_{1} \in L_{\mathrm{loc}}^{1}(I ; \mathbb{R})$, we say that $F$ is locally integrally bounded. 
Remark 1 If $\overline{D(A)}$ is separable, we can choose $N_{1}$ in Definition 6 independent of $(\tau, \xi) \in \mathcal{K}$ and, in this case, for each $(\tau, \xi) \in\left[\left(I \backslash N_{1}\right) \times X\right] \cap \mathcal{K}, F(\tau, \xi)$ is bounded.

Excepting the case when $K: I \rightsquigarrow X$ is constant, i.e., $K(t) \equiv C$, when $\mathcal{K}=I \times C$ is a cylindrical domain, one may happen that there would be no multi-function $F: \mathcal{K} \rightsquigarrow X$ such that $\mathcal{K}$ be $C^{0}$-viable with respect to $A+F$. See Example 2.1 in Necula, Popescu, Vrabie [18].

So, in order to get necessary and even necessary and sufficient conditions for the viability of a noncylindrical graph with respect to a given multi-functions, one has to consider merely a special class of graphs. This class of graphs, we are going to define precisely below, was considered for the first time by Necula [17].

Definition 7 Let $K: I \rightsquigarrow \overline{D(A)}$ be a multi-function. The graph, $\mathcal{K}$, of $K$ is said to be $A$ - $C^{0}$-viable by itself if for each $(\tau, \xi) \in \mathcal{K}$, there exist $T>\tau, \rho>0$ and $\ell_{2} \in L_{\mathrm{loc}}^{1}(I ; \mathbb{R})$, so that for each $(\widetilde{\tau}, \widetilde{\xi}) \in$ $([\tau, T) \times S(\xi, \rho)) \cap \mathcal{K}$, there exist $\widetilde{T} \in(\widetilde{\tau}, T]$ and a pair of functions, $(g, v) \in L^{1}([\widetilde{\tau}, \widetilde{T}] ; X) \times C([\widetilde{\tau}, \widetilde{T}] ; X)$, satisfying:

$$
\begin{aligned}
& \left(v_{1}\right) v(t)=u(t, \widetilde{\tau}, \widetilde{\xi}, g) \quad \text { for each } t \in[\widetilde{\tau}, \widetilde{T}] ; \\
& \left(v_{2}\right) \quad(t, v(t)) \in([\widetilde{\tau}, \widetilde{T}] \times S(\xi, \rho)) \cap \mathcal{K} \quad \text { for each } t \in[\widetilde{\tau}, \widetilde{T}] ; \\
& \left(v_{3}\right)\|g(s)\| \leq \ell_{2}(s) \quad \text { a.e. for } s \in[\widetilde{\tau}, \widetilde{T}] .
\end{aligned}
$$

By a simple solution issuing from $(\widetilde{\tau}, \widetilde{\xi}) \in([\tau, T) \times S(\xi, \rho)) \cap \mathcal{K}$ we mean a pair $(g, v)$ satisfying $\left(v_{1}\right)-\left(v_{3}\right)$.

Remark 2 In other words, the graph, $\mathcal{K}$, of $K: I \rightsquigarrow \overline{D(A)}$ is $A$ - $C^{0}$-viable by itself if and only if, for each $(\tau, \xi) \in \mathcal{K}$, there exist $T>\tau, \rho>0$ and $\ell_{2} \in L_{\text {loc }}^{1}(I ; \mathbb{R})$, so that $([\tau, T) \times S(\xi, \rho)) \cap \mathcal{K}$ is $C^{0}$-viable with respect to $A+G$, where the multi-function $G:([\tau, T) \times X) \cap \mathcal{K} \rightsquigarrow X$ is defined by

$$
G(t, \xi):=\left\{v \in X ;\|v\| \leq \ell_{2}(t)\right\},
$$

for each $(t, \xi) \in([\tau, T) \times X) \cap \mathcal{K}$.

Remark 3 (i) If $K: I \rightsquigarrow \overline{D(A)}$ is constant and $S(t) K \subseteq K$ for each $t \geq 0$, then $\mathcal{K}$ is $A$ - $C^{0}$-viable by itself.

(ii) If $\mathcal{K}$ is $C^{0}$-viable with respect to $A+F$, where $F: \mathcal{K} \rightsquigarrow X$ is some locally essentially bounded multi-function then, for each $(\tau, \xi) \in \mathcal{K}$, the function $G$, defined as in Remark 2 , with $\ell_{2}=\ell_{1}$, where $\ell_{1}$ is given by Definition 6, satisfies the conditions in Remark 6, and thus $\mathcal{K}$ is $A$ - $C^{0}$-viable by itself.

\section{Necessary conditions for viability}

Throughout, $\lambda$ denotes the Lebesgue measure on $\mathbb{R}$. First we recall

Definition 8 An m-dissipative operator $A: D(A) \rightsquigarrow X$ is of compact type if for each sequence $\left(f_{n}, u_{n}\right)_{n}$ in $L^{1}(\tau, T ; X) \times C([\tau, T] ; X)$ with $u_{n}$ a $C^{0}$-solution of the problem $u_{n}^{\prime}(t) \in A u_{n}(t)+f_{n}(t)$ on $[\tau, T]$ for $n=1,2, \ldots, \lim _{n} f_{n}=f$ weakly in $L^{1}(\tau, T ; X)$ and $\lim _{n} u_{n}=u$ strongly in $C([\tau, T] ; X)$, it follows that $u$ is a $C^{0}$-solution of the problem $u^{\prime}(t) \in A u(t)+f(t)$ on $[\tau, T]$.

A typical example of $m$-dissipative nonlinear operator of compact type is given by $\Delta \beta$ in $L^{1}(\Omega)$ with Dirichlet boundary conditions. See Diaz, Vrabie [13] and Cârjă, Necula, Vrabie [9], Theorem 1.7.9, p. 22.

The hypotheses we will use in the sequel are listed below. 
$\left(A_{1}\right) \quad A: D(A) \subseteq X \rightsquigarrow X$ is $m$-dissipative and $\{S(t): \overline{D(A)} \rightarrow \overline{D(A)} ; t \geq 0\}$ is the nonlinear semigroup of contractions generated by $A$;

$\left(A_{2}\right) \quad A: D(A) \subseteq X \rightsquigarrow X$ is $m$-dissipative and $\{S(t): \overline{D(A)} \rightarrow \overline{D(A)} ; t \geq 0\}$ is compact, i.e., $S(t)$ is compact for each $t>0$;

$\left(A_{3}\right) \quad A: D(A) \subseteq X \rightsquigarrow X$ is $m$-dissipative and of compact type;

$\left(F_{1}\right)$ the graph $\mathcal{K}$ is $A-C^{0}$-viable by itself;

$\left(F_{2}\right) \quad F$ has nonempty and closed values;

$\left(F_{3}\right) \quad F: \mathcal{K} \rightsquigarrow X$ is almost u.s.c.;

$\left(F_{4}\right) \quad F: \mathcal{K} \rightsquigarrow X$ is essentially locally bounded;

$\left(F_{5}\right) \quad F: \mathcal{K} \rightsquigarrow X$ is almost strongly-weakly u.s.c.;

( $\left.F_{6}\right)$ there exists a set $N \subseteq I$, with $\lambda(N)=0$, and such that for each $(\tau, \xi) \in[(I \backslash N) \times X] \cap \mathcal{K}$, we have $F(\tau, \xi) \in \mathcal{Q T}_{\mathcal{K}}^{A}(\tau, \bar{\xi})$.

Theorem 3 Let $\overline{D(A)}$ be separable. If $\left(A_{1}\right)$, and $\left(F_{3}\right)$ are satisfied, $F$ is nonempty-valued and locally integrally bounded, and $\mathcal{K}$ is $C^{0}$-viable with respect to $A+F$, then both $\left(F_{1}\right)$ and $\left(F_{6}\right)$ hold.

Proof. In view of (ii) in Remark 3, it remains to check out only $\left(F_{6}\right)$. Since $\overline{D(A)}$ is separable, and $F$ is locally integrally bounded, from Remark 1 , it follows that there exist a finite or countable set $\Gamma$, $\left(\tau_{i}, \xi_{i}\right)_{i \in \Gamma} \subseteq \mathcal{K},\left(\delta_{i}\right)_{i \in \Gamma} \subseteq(0, \infty),\left(\rho_{i}\right)_{i \in \Gamma} \subseteq(0, \infty),\left(\ell_{i}\right)_{i \in \Gamma} \subseteq L_{\text {loc }}^{1}(I ; \mathbb{R})$ and a negligible set $N \subseteq I$ such that $\mathcal{K} \subseteq \cup_{i \in \Gamma}\left(\tau_{i}-\delta_{i}, \tau_{i}+\delta_{i}\right) \times S\left(\xi_{i}, \rho_{i}\right)$ and, for each $i \in \Gamma$ and each $(t, u) \in\left(\left(\left(\tau_{i}-\delta_{i}, \tau_{i}+\delta_{i}\right) \backslash\right.\right.$ $\left.N) \times S\left(\xi_{i}, \rho_{i}\right)\right) \cap \mathcal{K}$, we have $\|F(t, u)\| \leq \ell_{i}(t)$.

From $\left(F_{3}\right)$ it follows that for each $n \geq 1$ it exists $I_{n} \subset I$ such that $\lambda\left(I \backslash I_{n}\right)<1 / n$ and $F$ is u.s.c. on $\left(I_{n} \times X\right) \cap \mathcal{K}$.

Let $E_{n} \subset I_{n}$ the set of all density points of $I_{n}$ which are also Lebesgue points for $\ell_{i}$, for all $i \in \Gamma$. Let $E=\left(\cup_{n \geq 1} E_{n}\right) \cap(I \backslash N)$. Obviously, $\lambda(I \backslash E)=0$.

Let $\tau \in E$ and $\xi \in K(\tau)$. We will show that $F(\tau, \xi) \in Q \mathcal{T} \mathcal{S}_{\mathcal{K}}^{A}(\tau, \xi)$.

Let $u:[\tau, T] \rightarrow \overline{D(A)}$ be a solution of (1). Hence there exists $f \in L^{1}(\tau, T ; X)$ such that $f(s) \in$ $F(s, u(s))$ a.e. $s \in[\tau, T]$ and $u=u(\cdot, \tau, \xi, f)$.

Since $\tau \in E$, there exists $n_{0} \in \mathbb{N}$ such that $\tau \in E_{n_{0}}$. Analogously, since $\mathcal{K} \subseteq \cup_{i \in \Gamma}\left(\tau_{i}-\delta_{i}, \tau_{i}+\delta_{i}\right) \times$ $S\left(\xi_{i}, \rho_{i}\right)$, there exists $i_{0} \in \Gamma$ such that $(\tau, \xi) \in\left(\tau_{i_{0}}-\delta_{i_{0}}, \tau_{i_{0}}+\delta_{i_{0}}\right) \times S\left(\xi_{i_{0}}, \rho_{i_{0}}\right)$. Let $\varepsilon>0$ be arbitrary but fixed and let $0<\delta<\delta_{i_{0}}$ be such that

$$
f(s) \in F(s, u(s)) \subset F(\tau, \xi)+D(0, \varepsilon),
$$

a.e. for $s \in[\tau, \tau+\delta] \cap E_{n_{0}}$ and $u(s) \in S\left(\xi_{i_{0}}, \rho_{i_{0}}\right)$ for all $s \in[\tau, \tau+\delta]$.

Let $\eta \in F(\tau, \xi)$ be fixed and

$$
\widetilde{f}(s)=\left\{\begin{array}{ll}
f(s) & \text { for } s \in[\tau, \tau+\delta] \cap E_{n_{0}} \\
\eta & \text { for } s \in[\tau, \tau+\delta] \backslash E_{n_{0}}
\end{array} .\right.
$$

Hence $\widetilde{f}(s) \in F(\tau, \xi)+D(0, \varepsilon)$ a.e. for $s \in[\tau, \tau+\delta]$.

Let $\bar{f}:[\tau, \tau+\delta] \rightarrow X$ countably valued such that $\|\bar{f}(s)-\widetilde{f}(s)\|<\varepsilon$ a.e. for $s \in[\tau, \tau+\delta]$. So, we have

$$
\bar{f}(s) \in F(\tau, \xi)+D(0,2 \varepsilon)
$$

a.e. for $s \in[\tau, \tau+\delta]$. 
Then, there exist two countably valued functions $g:[\tau, \tau+\delta] \rightarrow F(\tau, \xi)$ and $r:[\tau, \tau+\delta] \rightarrow D(0,2 \varepsilon)$ such that

$$
\bar{f}(s)=g(s)+r(s)
$$

a.e. for $s \in[\tau, \tau+\delta]$. Hence $g, r \in L^{1}(\tau, \tau+\delta ; X)$.

Since $u(\tau+h) \in K(\tau+h),\|g(s)-\widetilde{f}(s)\| \leq 3 \varepsilon$ a.e. for $s \in[\tau, \tau+\delta]$, using Corollary 1, we deduce that for each $0<h<\delta$

$$
\begin{aligned}
\frac{1}{h} \operatorname{dist} & \left(u\left(\tau+h, \tau, \xi, \mathcal{F}_{F(\tau, \xi)}\right), K(\tau+h)\right) \leq \frac{1}{h}\|u(\tau+h, \tau, \xi, g)-u(\tau+h, \tau, \xi, f)\| \\
& \leq \frac{1}{h} \int_{\tau}^{\tau+h}\|g(s)-f(s)\| \mathrm{d} s \\
& \leq \frac{1}{h} \int_{\tau}^{\tau+h}\|g(s)-\widetilde{f}(s)\| \mathrm{d} s+\frac{1}{h} \int_{\tau}^{\tau+h}\|\widetilde{f}(s)-f(s)\| \mathrm{d} s \\
& \leq 3 \varepsilon+\frac{1}{h} \int_{[\tau, \tau+h] \backslash E_{n_{0}}}\|f(s)-\eta\| \mathrm{d} s \quad\left\|\ell_{i_{0}}(s)\right\| \mathrm{d} s+\frac{1}{h} \int_{[\tau, \tau+h] \backslash E_{n_{0}}} \| \eta \mathrm{d} s \\
& \leq 3 \varepsilon+\frac{1}{h} \int_{[\tau, \tau+h] \backslash E_{n_{0}}}^{\tau}\left\|\frac{1}{h} \int_{\tau}^{\tau+h}\right\| \ell_{i_{0}}(s)-\ell_{i_{0}}(\tau) \| \mathrm{d} s+\left(\left\|\ell_{i_{0}}(\tau)\right\|+\|\eta\|\right)\left(1-\frac{\lambda\left([\tau, \tau+h] \cap E_{n_{0}}\right)}{\lambda([\tau, \tau+h])}\right) .
\end{aligned}
$$

Passing to limsup in the inequality above and recalling that $\tau$ is both a density point and a Lebesgue point for $\ell_{i_{0}}$, we get

$$
\limsup _{h \downarrow 0} \frac{1}{h} \operatorname{dist}\left(u\left(\tau+h, \tau, \xi, \mathcal{F}_{F(\tau, \xi)}\right), K(\tau+h)\right) \leq 3 \varepsilon .
$$

As $\varepsilon>0$ was arbitrary, we deduce $\left(F_{6}\right)$.

In fact, we have proved a stronger result, i.e.,

Theorem 4 Let $\overline{D(A)}$ be separable. If $\left(A_{1}\right)$, and $\left(F_{3}\right)$ are satisfied, $F$ is nonempty-valued and locally integrally bounded, and $\mathcal{K}$ is $C^{0}$-viable with respect to $A+F$, then $\left(F_{1}\right)$ holds and there exists a set $N \subseteq I$, with $\lambda(N)=0$, and such that for each $(\tau, \xi) \in((I \backslash N) \times X) \cap \mathcal{K}$, we have

$$
\lim _{h \downarrow 0} \frac{1}{h} \operatorname{dist}\left(u\left(\tau+h, \tau, \xi, \mathcal{F}_{F(\tau, \xi)}\right) ; K(\tau+h)\right)=0 .
$$

\section{Sufficient conditions for viability}

Definition 9 We say that the graph $\mathcal{K}$ is:

(i) locally closed from the left if for each $(\tau, \xi) \in \mathcal{K}$ there exist $T>\tau$ and $\rho>0$ such that, for each $\left(\tau_{n}, \xi_{n}\right) \in([\tau, T] \times D(\xi, \rho)) \cap \mathcal{K}$, with $\left(\tau_{n}\right)_{n}$ nondecreasing, $\lim _{n} \tau_{n}=\widetilde{\tau}$ and $\lim _{n} \xi_{n}=\widetilde{\xi}$, we have $(\widetilde{\tau}, \widetilde{\xi}) \in \mathcal{K}$

(ii) closed from the left if for each $\left(\tau_{n}, \xi_{n}\right) \in \mathcal{K}$, with $\left(\tau_{n}\right)_{n}$ nondecreasing, $\lim _{n} \tau_{n}=\widetilde{\tau}$ and $\lim _{n} \xi_{n}=$ $\widetilde{\xi}$, we have $(\widetilde{\tau}, \widetilde{\xi}) \in \mathcal{K}$.

Theorem 5 Let $\mathcal{K}$ be locally closed from the left and let $F: \mathcal{K} \rightsquigarrow X$ be nonempty, convex and weakly compact valued. If $\left(A_{1}\right),\left(A_{2}\right),\left(A_{3}\right),\left(F_{1}\right),\left(F_{2}\right),\left(F_{4}\right)$ and $\left(F_{5}\right)$ are satisfied, then a sufficient condition in order that $\mathcal{K}$ be $C^{0}$-viable with respect to $A+F$ is $\left(F_{6}\right)$. If, instead of $\left(F_{5}\right)$, the stronger condition $\left(F_{3}\right)$ is satisfied, then $\left(F_{6}\right)$ is also necessary in order that $\mathcal{K}$ be $C^{0}$-viable with respect to $A+F$. 
The necessity follows from Theorem 3 by observing that $\left(A_{2}\right)$ implies the separability of $\overline{D(A)}$. This separability result is a straightforward extension of its linear counterpart in Vrabie [26, Theorem 6.2.2, p. 136]. The sufficiency, which is by far the most interesting part of Theorem 5, will be proved later.

From Theorem 5, by a slight extension of Brezis-Browder Ordering Principle [7], i.e. Theorem 2.1.1, p. 30 in Cârjă, Necula, Vrabie [9], we deduce the global $C^{0}$-viability result below.

Theorem 6 Let $\mathcal{K}$ be closed from the left and let $F: \mathcal{K} \rightsquigarrow X$ be nonempty, convex and weakly compact valued. If $\left(A_{1}\right),\left(A_{2}\right),\left(A_{3}\right),\left(F_{1}\right),\left(F_{2}\right),\left(F_{4}\right)$ and $\left(F_{5}\right)$ are satisfied, then a sufficient condition in order that $\mathcal{K}$ be globally $C^{0}$-viable with respect to $A+F$ is $\left(F_{6}\right)$. If, instead of $\left(F_{5}\right)$, the stronger condition $\left(F_{3}\right)$ is satisfied, then $\left(F_{6}\right)$ is also necessary.

The next lemma is the main step through the proof of Theorem 5.

Lemma 1 Let $X$ be a real Banach space, $A: D(A) \subseteq X \rightsquigarrow X$ an m-dissipative operator, I a nonempty and open from the right interval, $K: I \rightsquigarrow \overline{D(A)}$ a multi-function with locally closed from the left graph and $F: \mathcal{K} \rightsquigarrow X$ a nonempty-valued, locally essentially bounded multi-function. Let us assume that $\left(A_{1}\right)$, $\left(F_{1}\right)\left(F_{2}\right),\left(F_{4}\right)$, and $\left(F_{6}\right)$ are satisfied. Let $(\tau, \xi) \in \mathcal{K}$ and let $Z=N_{1} \cup N$, where $N_{1}$ and $N$ are the negligible sets in $\left(F_{4}\right)$ and in $\left(F_{6}\right)$.

Let $\rho>0$ and $\widetilde{T}>\tau$ be such that $([\tau, \widetilde{T}] \times D(\xi, \rho)) \cap \mathcal{K}$ is closed from the left, $F$ is a.e. bounded by $\ell_{1} \in L_{\mathrm{loc}}^{\infty}(I ; \mathbb{R})$ on $([\tau, \widetilde{T}] \times D(\xi, \rho)) \cap \mathcal{K}$-see Definition 6 and let $\ell_{2} \in L_{\mathrm{loc}}^{1}(I ; \mathbb{R})$ be given by Definition 7 .

Then, for each $\varepsilon \in(0,1)$ and each open set $\mathcal{O} \subseteq I$, with $Z \subseteq \mathcal{O}$, there exist $T \in(\tau, \widetilde{T}]$ and three functions: $\alpha:[\tau, T] \rightarrow[\tau, T]$ nondecreasing and right continuous, $f:[\tau, T] \rightarrow X$ measurable and $v:[\tau, T] \rightarrow X$ continuous satisfying:

(i) $t-\varepsilon \leq \alpha(t) \leq t \quad$ for all $t \in[\tau, T], \alpha(T)=T$;

(ii) for each $t \in[\tau, T]$ for which $\alpha(t) \in \mathcal{O}$ it follows that $[\alpha(t), t] \subseteq \mathcal{O}$;

(iii) $v(\alpha(t)) \in D(\xi, \rho) \cap K(\alpha(t))$ for all $t \in[\tau, T]$;

(iv) $f(t) \in F(\alpha(t), v(\alpha(t)))$ for each $t \in[\tau, T] \backslash \mathcal{O}$;

(v) $\|f(t)\| \leq \ell(t) \quad$ a.e. for $t \in[\tau, T]$, with $\ell(t)=\max \left\{\ell_{1}(t), \ell_{2}(t)\right\}$, where $\ell_{1} \in L_{\mathrm{loc}}^{\infty}(I ; \mathbb{R})$ is as in Definition 6 and $\ell_{2} \in L_{\mathrm{loc}}^{1}(I ; \mathbb{R})$ as in Definition 7;

(vi) $v(\tau)=\xi$ and $\|v(t)-u(t, \alpha(s), v(\alpha(s)), f)\| \leq(t-\alpha(s)) \varepsilon \quad$ for all $t, s \in[\tau, T], \tau \leq s \leq t \leq T$;

(vii) $\|v(t)-v(\alpha(t))\| \leq \varepsilon \quad$ for all $t \in[\tau, T]$;

(viii) $\sup _{t \in[\tau, T]}\|S(t-\tau) \xi-\xi\|+\int_{\tau}^{T} \ell(s) \mathrm{d} s+T-\tau \leq \rho$.

Definition 10 Let $(\tau, \xi) \in \mathcal{K}, \varepsilon \in(0,1)$ and $\mathcal{O} \subseteq I$ a nonempty and open set with $Z \subseteq \mathcal{O}$. A triplet $(\alpha, f, v)$ satisfying (i)-(viii) is called an $(\varepsilon, \mathcal{O})$-approximate $C^{0}$-solution of $(1)$.

We can now proceed to the proof of Lemma 1.

Proof OF Lemma 1 Let $(\tau, \xi) \in \mathcal{K}$ be arbitrary and choose $\rho>0$ and $T>\tau$ such that

$$
([\tau, T] \times D(\xi, \rho)) \cap \mathcal{K}
$$

is closed from the left. This is always possible because $\mathcal{K}$ is locally closed. Next, diminishing $T>\tau$ if necessary, we may assume that (viii) holds.

We first prove that the conclusion of Lemma 1 remains true if we replace $T$ as above with a possible smaller number $\tau+\delta$ with $\delta \in(0, T-\tau]$ which, at this stage, is allowed to depend on $\varepsilon \in(0,1)$. Then, by using the extension of Brezis-Browder Ordering Principle [7], i.e. Theorem 2.1.1, p. 30 in Cârjă, Necula, Vrabie [9], we will prove that we can take $\tau+\delta=T$ independent of $\varepsilon$.

Let $\varepsilon \in(0,1)$ be arbitrary. We distinguish between the following two complementary cases. 
Case 1. If $\tau \in \mathcal{O}$, we take $\alpha:[\tau, \tau+\delta] \rightarrow[\tau, \tau+\delta]$ defined by $\alpha(t)=\tau$ for $t \in[\tau, \tau+\delta), \alpha(\tau+\delta)=\tau+\delta$. In order to define $f$ and $v$, let us recall that there exists a simple solution $(g, v)$ issuing from $(\tau, \xi)$, defined on $[\tau, \tau+\delta]$. Let $(g, v)$ be such a simple solution, and let us define $f(s)=g(s)$ a.e. for $s \in[\tau, \tau+\delta]$. Obviously (i) and (iii)-(vi) are satisfied and, taking into account that $\mathcal{O}$ is open and $v$ is continuous, diminishing $\delta$ if necessary, we conclude that (ii) and (vii) are satisfied too.

Case 2. If $\tau \notin \mathcal{O}$ then $\tau \notin Z$, then $F(\tau, \xi) \in Q \mathcal{O} \mathcal{S}_{K}^{A}(\tau, \xi)$, and therefore there exist $f \in \mathcal{F}_{F(\tau, \xi)}, \delta \in(0, \varepsilon)$ and $p \in X$ with $\|p\| \leq \varepsilon$ such that

$$
u(\tau+\delta, \tau, \xi, f)+\delta p \in K(\tau+\delta) .
$$

We recall that $\mathcal{F}_{F(\tau, \xi)}=\left\{f \in L^{1}\left(\mathbb{R}_{+} ; X\right) ; f(s) \in F(\tau, \xi)\right.$ a.e. for $\left.s \in \mathbb{R}_{+}\right\}$. With $f$ as above, let us define $\alpha:[\tau, \tau+\delta] \rightarrow[\tau, \tau+\delta]$ and $v:[\tau, \tau+\delta] \rightarrow X$ by $\alpha(t)=\tau$ for $t \in[\tau, \tau+\delta)$, $\alpha(\tau+\delta)=\tau+\delta$, and respectively by

$$
v(t)=u(t, \tau, \xi, f)+(t-\tau) p
$$

for each $t \in[\tau, \tau+\delta]$.

Let us observe that the functions $\alpha, f$ and $v$ satisfy (i)-(v) with $T=\tau+\delta$. Clearly, $v(\tau)=\xi$. Moreover, since $\|p\| \leq \varepsilon$, we deduce

$$
\|v(t)-u(t, \alpha(s), v(\alpha(s)), f)\|=\|v(t)-u(t, \tau, v(\tau), f)\|=(t-\tau)\|p\| \leq(t-\alpha(s)) \varepsilon
$$

for all $\tau \leq s \leq t \leq \tau+\delta$. Thus (vi) is also satisfied. Next, diminishing $\delta>0$ and redefining $\alpha$ if necessary, we get

$$
\begin{aligned}
\|v(t)-v(\alpha(t))\| & =\|v(t)-v(\tau)\| \\
& \leq\|u(t, \tau, \xi, f)-\xi\|+(t-\tau)\|p\| \\
& \leq\|S(t-\tau) \xi-\xi\|+\int_{\tau}^{t}\|f(s)\| \mathrm{d} s+(t-\tau) \varepsilon \\
& \leq \sup _{t \in[\tau, \tau+\delta]}\|S(t-\tau) \xi-\xi\|+\int_{\tau}^{\tau+\delta} \ell(s) \mathrm{d} s+\delta \leq \varepsilon
\end{aligned}
$$

for each $t \in[\tau, \tau+\delta)$, and thus (vii) is also satisfied.

Next, we will show that there exists at least one triplet $(\alpha, f, v)$ satisfying (i)-(viii) on $[\tau, T]$. We shall use the extension of Brezis-Browder Ordering Principle [7], i.e. Theorem 2.1.1, p. 30 in Cârjă, Necula, Vrabie [9], as follows. Let $\mathcal{S}$ be the set of all triplets $(\alpha, f, v)$, defined on $[\tau, \mu]$, with $\tau<\mu \leq T$ and satisfying (i)-(viii) with $\mu$ instead of $T$. This set is clearly nonempty, as we have already proved. On $\mathcal{S}$ we introduce a partial order $\preceq$ as follows. We say that

$$
\left(\alpha_{1}, f_{1}, v_{1}\right) \preceq\left(\alpha_{2}, f_{2}, v_{2}\right)
$$

if $\mu_{1} \leq \mu_{2}$ and $\alpha_{1}(s)=\alpha_{2}(s), f_{1}(s)=f_{2}(s)$ and $v_{1}(s)=v_{2}(s)$ for each $s \in\left[\tau, \mu_{1}\right]$.

Let us define the function $\mathcal{N}: \mathcal{S} \rightarrow \mathbb{R}$ by

$$
\mathcal{N}(\alpha, f, v)=\mu .
$$

It is clear that $\mathcal{N}$ is increasing on $\mathcal{S}$. Let us now take an increasing sequence

$$
\left(\left(\alpha_{j}, f_{j}, v_{j}\right)\right)_{j}
$$

in $\mathcal{S}$ and let us show that it is bounded from above in $\mathcal{S}$. We define an upper bound as follows. First, set

$$
\mu^{*}=\sup \left\{\mu_{j} ; j \in \mathbb{N}\right\} .
$$


If $\mu^{*}=\mu_{j}$ for some $j \in \mathbb{N},\left(\alpha_{j}, f_{j}, v_{j}\right)$ is clearly an upper bound. If $\mu_{j}<\mu^{*}$ for each $j \in \mathbb{N}$, let us define

$$
\alpha(t)=\alpha_{j}(t), \quad f(t)=f_{j}(t), \quad v(t)=v_{j}(t)
$$

for $j \in \mathbb{N}$ and every $t \in\left[\tau, \mu_{j}\right]$. To extend $\alpha, f$ and $v$ to $t=\mu^{*}$, we proceed as follows.

First, we extend $f$ at $\mu^{*}$ by setting $f\left(\mu^{*}\right)=\eta$, where $\eta \in X$ is arbitrary but fixed.

Second, by (iv) and (v), it follows that $f \in L^{1}\left(\tau, \mu^{*} ; X\right)$ and therefore, for each $j \in \mathbb{N}$, the function $u\left(\cdot, \mu_{j}, v\left(\mu_{j}\right), f\right):\left[\mu_{j}, \mu^{*}\right] \rightarrow \overline{D(A)}$ is continuous.

To extend $v$ to $\mu^{*}$, it suffices to show that there exists $\lim _{t \uparrow \mu^{*}} v(t)$. To this aim, let us observe that, in view of (vi), we have

$$
\begin{aligned}
\|v(t)-v(\widetilde{t})\| \leq & \left\|v(t)-u\left(t, \mu_{j}, v\left(\mu_{j}\right), f\right)\right\| \\
& \quad+\left\|u\left(t, \mu_{j}, v\left(\mu_{j}\right), f\right)-u\left(\widetilde{t}, \mu_{j}, v\left(\mu_{j}\right), f\right)\right\|+\left\|u\left(\widetilde{t}, \mu_{j}, v\left(\mu_{j}\right), f\right)-v(\widetilde{t})\right\| \\
\leq & \left(t-\mu_{j}\right) \varepsilon+\left\|u\left(t, \mu_{j}, v\left(\mu_{j}\right), f\right)-u\left(\widetilde{t}, \mu_{j}, v\left(\mu_{j}\right), f\right)\right\|+\left(\widetilde{t}-\mu_{j}\right) \varepsilon \\
\leq & \left(\mu^{*}-\mu_{j}\right) \varepsilon+\left\|u\left(t, \mu_{j}, v\left(\mu_{j}\right), f\right)-u\left(\widetilde{t}, \mu_{j}, v\left(\mu_{j}\right), f\right)\right\|+\left(\mu^{*}-\mu_{j}\right) \varepsilon,
\end{aligned}
$$

for each $j \in \mathbb{N}$, each $t \geq \mu_{j}$ and each $\widetilde{t} \geq \mu_{j}$. Since $\lim _{j} \mu_{j}=\mu^{*}$ and $u\left(\cdot, \mu_{j}, v\left(\mu_{j}\right)\right)$ is continuous at $t=\mu^{*}$, we conclude that $t \mapsto v(t)$ satisfies the Cauchy necessary and sufficient condition for the existence of the limit at $t=\mu^{*}$. Indeed, let $\varepsilon^{\prime}>0$ be arbitrary and let us fix $j \in \mathbb{N}$ such that $\left(\mu^{*}-\mu_{j}\right) \varepsilon \leq \varepsilon^{\prime} / 3$. Next, let us fix $\delta\left(\varepsilon^{\prime}\right)>0$ such that, for each $t, \widetilde{t} \in\left[\mu_{j}, \mu^{*}\right)$ with $\mu^{*}-t \leq \delta\left(\varepsilon^{\prime}\right)$ and $\mu^{*}-\widetilde{t} \leq \delta\left(\varepsilon^{\prime}\right)$, we have $\left\|u\left(t, \mu_{j}, v\left(\mu_{j}\right), f\right)-u\left(\widetilde{t}, \mu_{j}, v\left(\mu_{j}\right), f\right)\right\| \leq \varepsilon^{\prime} / 3$. Thus, for each $t$ and $\widetilde{t}$ as above, we have $\|v(t)-v(\widetilde{t})\| \leq \varepsilon^{\prime}$, as claimed. So, we can extend $v$, by continuity, to the whole interval $\left[\tau, \mu^{*}\right]$. Finally, we define $\alpha\left(\mu^{*}\right)=\mu^{*}$.

Since $v\left(\mu_{m}\right) \in D(\xi, \rho) \cap K\left(\mu_{m}\right)$, for each $m \in \mathbb{N}$, and the latter is closed from the left, we deduce that $v\left(\mu^{*}\right) \in D(\xi, \rho) \cap K\left(\mu^{*}\right)$. At this point, let us observe that, if necessary, i.e., if $\mu^{*} \notin \mathcal{O}$, we have to redefine $f\left(\mu^{*}\right)=\eta$ by choosing $\eta \in F\left(\mu^{*}, v\left(\mu^{*}\right)\right)$, in order that (iv) be satisfied. This is always possible because $f$ is supposed to be in $L^{1}\left(\tau, \mu^{*} ; X\right)$. Hence, $(\alpha, f, v)$ satisfies (i)-(iv). Next, we may easily verify that $(\alpha, f, v)$ satisfies (v)-(viii) and so, it is an upper bound for $\left(\left(\alpha_{j}, f_{j}, v_{j}\right)\right)_{j}$. Consequently the set $\mathcal{S}$ endowed with the partial order $\preceq$ and the function $\mathcal{N}$ satisfy the hypotheses of the extension of Brezis-Browder Ordering Principle [7], i.e. Theorem 2.1.1, p. 30 in Cârjă, Necula, Vrabie [9]. Accordingly, there exists at least one $\mathcal{N}$-maximal element $\left(\alpha_{\nu}, f_{\nu}, v_{\nu}\right)$ in $\mathcal{S}$, i.e., an element such that, if $\left(\alpha_{\nu}, f_{\nu}, v_{\nu}\right) \preceq\left(\alpha_{\sigma}, f_{\sigma}, v_{\sigma}\right)$ then $\nu=\sigma$.

We next show that $\nu=T$, where $T$ satisfies (viii). We prove this by contraposition, i.e., we show that an element $\left(\alpha_{\nu}, f_{\nu}, v_{\nu}\right)$ in $\mathcal{S}$ with $\nu<T$ is not $\mathcal{N}$-maximal. So, let us assume that $\nu<T$ and let $\xi_{\nu}=v_{\nu}(\nu)=v_{\nu}\left(\alpha_{\nu}(\nu)\right)$ which, by (iii), belongs to $D(\xi, \rho) \cap K(\nu)$. In view of (v) and (vi), we have

$$
\begin{aligned}
\left\|\xi_{\nu}-\xi\right\| & \leq\|S(\nu-\tau) \xi-\xi\|+\left\|u\left(\nu, \tau, \xi, f_{\nu}\right)-S(\nu) \xi\right\|+\left\|v_{\nu}(\nu)-u\left(\nu, \tau, \xi, f_{\nu}\right)\right\| \\
& \leq\|S(\nu-\tau) \xi-\xi\|+\int_{\tau}^{\nu}\left\|f_{\nu}(s)\right\| \mathrm{d} s+(\nu-\tau) \varepsilon \\
& \leq \sup _{0 \leq t \leq \nu-\tau}\|S(t) \xi-\xi\|+\int_{\tau}^{\nu} \ell(s) \mathrm{d} s+(\nu-\tau) \varepsilon .
\end{aligned}
$$

Recalling that $\nu<T$ and $\varepsilon<1$, from (viii), we get

$$
\left\|\xi_{\nu}-\xi\right\|<\rho .
$$

At this point we act as at the beginning of the proof with $\nu$ instead of $\tau$ and with $\xi_{\nu}$ instead of $\xi$. So, distinguish between the following two complementary cases.

Case 1. If $\nu \in \mathcal{O}$, we take $\alpha:[\tau, \nu+\delta] \rightarrow[\tau, \nu+\delta]$ defined by

$$
\alpha_{\nu+\delta}(t)= \begin{cases}\alpha_{\nu}(t) & \text { if } t \in[\tau, \nu] \\ \nu & \text { if } t \in(\nu, \nu+\delta) \\ \nu+\delta & \text { if } t=\nu+\delta,\end{cases}
$$


In order to define $f_{\nu+\delta}$ and $v_{\nu+\delta}$, let us recall that there exists a simple solution $(g, v)$ issuing from $\left(\nu, \xi_{\nu}\right)$, defined on $[\nu, \nu+\delta]$. Let $(g, v)$ be such a simple solution, and let us define

$$
f_{\nu+\delta}(t)= \begin{cases}f_{\nu}(t) & \text { if } t \in[\tau, \nu] \\ g(t) & \text { if } t \in(\nu, \nu+\delta]\end{cases}
$$

and

$$
v_{\nu+\delta}(t)= \begin{cases}v_{\nu}(t) & \text { if } t \in[\tau, \nu] \\ v(t) & \text { if } t \in(\nu, \nu+\delta]\end{cases}
$$

One may easily see that (i) and (iii)-(vi) are satisfied and, taking into account that $\mathcal{O}$ is open and $v$ is continuous, diminishing $\delta$ if necessary, we conclude that (ii), (vii) and (viii) are satisfied too.

Case 2. If $\nu \notin \mathcal{O}$ then $\nu \notin Z$, then $F(\nu, \xi) \in Q \mathcal{T} \mathcal{S}_{K}^{A}(\nu, \xi)$. So, from (6), we infer that there exist $f \in \mathcal{F}_{F\left(\nu, \xi_{\nu}\right)}, \delta \in(0, \varepsilon]$ with $\nu+\delta \leq T$ and $p \in X$ satisfying $\|p\| \leq \varepsilon$, such that

$$
u\left(\nu+\delta, \nu, \xi_{\nu}, f\right)+\delta p \in D(\xi, \rho) \cap K(\nu+\delta)
$$

Let us define $\alpha_{\nu+\delta}:[\tau, \nu+\delta] \rightarrow[\tau, \nu+\delta], f_{\nu+\delta}:[\tau, \nu+\delta] \rightarrow X$ and $v_{\nu+\delta}:[\tau, \nu+\delta] \rightarrow X$ by

$$
\begin{aligned}
& \alpha_{\nu+\delta}(t)= \begin{cases}\alpha_{\nu}(t) & \text { if } t \in[\tau, \nu] \\
\nu & \text { if } t \in(\nu, \nu+\delta) \\
\nu+\delta & \text { if } t=\nu+\delta,\end{cases} \\
& f_{\nu+\delta}(t)= \begin{cases}f_{\nu}(t) & \text { if } t \in[\tau, \nu] \\
f(t) & \text { if } t \in(\nu, \nu+\delta],\end{cases}
\end{aligned}
$$

and

$$
v_{\nu+\delta}(t)= \begin{cases}v_{\nu}(t) & \text { if } t \in[\tau, \nu] \\ u\left(t, \nu, \xi_{\nu}, f_{\nu+\delta}\right)+(t-\nu) p & \text { if } t \in(\nu, \nu+\delta] .\end{cases}
$$

Since $v_{\nu+\delta}(\nu+\delta) \in D(\xi, \rho) \cap K(\nu+\delta),\left(\alpha_{\nu+\delta} f_{\nu+\delta}, v_{\nu+\delta}\right)$, it follows that satisfies (i)-(v), with $T$ replaced by $\nu+\delta$.

To check (vi) we consider the complementary cases: $s \leq t \leq \nu, \nu<s \leq t$ and $s \leq \nu \leq t$.

Clearly (vi) holds for each $t, s$ satisfying $s \leq t \leq \nu$. If $\nu<s \leq t$, we have

$$
\begin{aligned}
\| v_{\nu+\delta}(t)-u & \left(t, \alpha_{\nu+\delta}(s), v_{\nu+\delta}\left(\alpha_{\nu+\delta}(s)\right), f_{\nu+\delta}\right) \| \\
= & \left\|u\left(t, \nu, \xi_{\nu}, f_{\nu+\delta}\right)+(t-\nu) p-u\left(t, \nu, \xi_{\nu}, f_{\nu+\delta}\right)\right\| \\
\leq & (t-\nu) \varepsilon=\left(t-\alpha_{\nu+\delta}(s)\right) \varepsilon .
\end{aligned}
$$

Let now $s<\nu \leq t$, and let us observe that, by virtue of the evolution property (3) and of (vi) (which is valid on both $[\tau, \nu]$ and $[\nu, \nu+\delta]$, we have

$$
\begin{aligned}
v_{\nu+\delta}(t) & -u\left(t, \alpha_{\nu+\delta}(s), v_{\nu+\delta}\left(\alpha_{\nu+\delta}(s)\right), f_{\nu+\delta}\right) \\
& =u\left(t, \nu, v_{\nu+\delta}(\nu), f_{\nu+\delta}\right)+(t-\nu) p-u\left(t, \nu, u\left(\nu, \alpha_{\nu+\delta}(s), v_{\nu+\delta}\left(\alpha_{\nu+\delta}(s)\right), f_{\nu+\delta}\right), f_{\nu+\delta}\right) .
\end{aligned}
$$

Therefore

$$
\begin{aligned}
\| v_{\nu+\delta}(t)- & u\left(t, \alpha_{\nu+\delta}(s), v_{\nu+\delta}\left(\alpha_{\nu+\delta}(s)\right), f_{\nu+\delta}\right) \| \\
& \leq\left\|v_{\nu+\delta}(\nu)-u\left(\nu, \alpha_{\nu+\delta}(s), v_{\nu+\delta}\left(\alpha_{\nu+\delta}(s)\right), f_{\nu+\delta}\right)\right\|+(t-\nu)\|p\| \\
& \leq\left(\nu-\alpha_{\nu+\delta}(s)\right) \varepsilon+(t-\nu) \varepsilon \\
& =\left(t-\alpha_{\nu+\delta}(s)\right) \varepsilon
\end{aligned}
$$


which proves (vi).

Similarly, diminishing $\delta$ if necessary and redefining the functions $\alpha, f$ and $v$, we deduce that (vii) and (viii) are satisfied. So $\left(\alpha_{\nu+\delta}, f_{\nu+\delta}, v_{\nu+\delta}\right) \in \mathcal{S}$,

$$
\left(\alpha_{\nu}, f_{\nu}, v_{\nu}\right) \preceq\left(\alpha_{\nu+\delta}, f_{\nu+\delta}, v_{\nu+\delta}\right)
$$

and $\nu<\nu+\delta$. Hence $\left(\alpha_{\nu}, f_{\nu}, v_{\nu}\right)$ is not $\mathcal{N}$-maximal, and this completes the proof of Lemma 1.

Remark 4 Under the general hypotheses of Lemma 1, for each $\gamma>\tau$, we can diminish both $\rho>0$ and $T>\tau$, such that $T<\gamma, \rho<\gamma-\tau$ and all the conditions (i)-(viii) in Lemma 1 be satisfied.

\section{Proof of Theorem 5}

Proof of THEOREM 5 Since the necessity follows from Theorem 3, we will confine ourselves only to the proof of the sufficiency.

Let $Z \subseteq \mathbb{R}$ be a negligible set including the negligible set $N_{1}$ appearing in Definition 6 and the negligible set $N$ in $\left(F_{6}\right)$. Let $\rho>0$ and $T>\tau$ and $\ell$ be as in Lemma 1. Let $\varepsilon_{n} \in(0,1)$, with $\varepsilon_{n} \downarrow 0$, let $\left(\mathcal{O}_{n}\right)_{n \geq 1} \subseteq \mathbb{R}$ be a sequence of open sets such that:

(a) $Z \subseteq \mathcal{O}_{n}$ for each $n \in \mathbb{N}, n \geq 1$;

(b) $\mathcal{O}_{n+1} \subseteq \mathcal{O}_{n}$ and $\lambda\left([\tau, T] \cap \mathcal{O}_{n}\right) \leq \varepsilon_{n}$ for each $n \in \mathbb{N}, n \geq 1$;

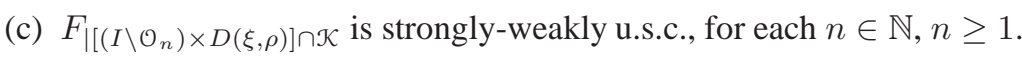

Let $\left(\left(a_{n}, f_{n}, u_{n}\right)\right)_{n}$ be a sequence of $\left(\varepsilon_{n}, \mathcal{O}_{n}\right)$-approximate solutions of $(1)$, sequence given by Lemma 1. Clearly

$$
\lim _{n} a_{n}(s)=s
$$

uniformly for $s \in[\tau, T)$.

In view of (vi) in Lemma 1, we have

$$
u_{n}(t)=u\left(t, \tau, \xi, f_{n}\right)+w_{n}(t)
$$

for each $n \in \mathbb{N}$ and $t \in[\tau, T]$, where $\lim _{n} w_{n}(t)=0$ uniformly for $t \in[\tau, T]$. We will show that, on a subsequence at least, $\left(u_{n}\right)_{n}$ is uniformly convergent on $[\tau, T]$ to some function $u$ which will turn out to be a $C^{0}$-solution for the problem (1).

To do this, it suffices to show that the sequence $\left(u\left(\cdot, \tau, \xi, f_{n}\right)\right)_{n}$ is uniformly convergent on $[\tau, T]$ to some function $u$.

Since $\left\|f_{n}(t)\right\| \leq \ell(t)$ for each $n \in \mathbb{N}$ and a.e. for $t \in[\tau, T]$, and the semigroup generated by $A$ is compact, by virtue of Baras' Theorem 2.3.3, p. 47, in Vrabie [25], we conclude that $\left(u_{n}\right)_{n}$ has at least one uniformly convergent subsequence to some function $u$. But $a_{n}(t) \uparrow t$ and $\lim _{n} u_{n}\left(a_{n}(t)\right)=u(t)$, uniformly for $t \in[\tau, T)$, and hence, for each $k \geq 1$, the set

$$
C_{k}=\overline{\left\{\left(a_{n}(t), u_{n}\left(a_{n}(t)\right)\right) ; n \geq k, t \in[\tau, T)\right\}}
$$

is compact. Since $F$ is strongly-weakly u.s.c. and has weakly compact values, by Lemma 2.6.1, p. 47, in Cârjă, Necula, Vrabie [9], it follows that, for each $k \geq 1$, the set

$$
B_{k}:=\overline{\operatorname{conv}}\left(\bigcup_{n \geq k} \bigcup_{t \in[\tau, T] \backslash \mathcal{O}_{k}} F\left(a_{n}(t), u_{n}\left(a_{n}(t)\right)\right)\right)
$$


is weakly compact. Using again the fact that $\left\|f_{n}(t)\right\| \leq \ell(t)$ for each $n \in \mathbb{N}$ and a.e. for $t \in[\tau, T]$, where $\ell \in L^{1}(\tau, T ; \mathbb{R})$, recalling that $B_{k}$ is weakly compact and $\lim _{k} \lambda\left(\mathcal{O}_{k}\right)=0$, by Diestel's Theorem 1.3.8, p. 10, in Cârjă, Necula, Vrabie [9], it follows that, on a subsequence at least, $\lim _{n} f_{n}=f$ weakly in $L^{1}(\tau, T ; X)$. By (ii) in Lemma 1 , for each $k \geq 1$ there exists $n(k) \in \mathbb{N}$ so that, for each $n \geq n(k) \geq k$, we have $a_{n}(s) \in[\tau, T] \backslash \mathcal{O}_{k}$ a.e. for $s \in[\tau, T] \backslash \mathcal{O}_{k}$. As $\lim _{n} u_{n}(t)=u(t)$ uniformly for $t \in[\tau, T], \lim _{n} f_{n}=f$ weakly in $L^{1}(\tau, T ; X), f_{n}(s) \in F\left(a_{n}(s), u_{n}\left(a_{n}(s)\right)\right)$ a.e. for $s \in[\tau, T] \backslash \mathcal{O}_{k}$, and $F_{\mid\left[\left(I \backslash \mathcal{O}_{k}\right) \times D(\xi, \rho)\right] \cap \mathcal{K}}$ is strongly-weakly u.s.c., from Theorem 3.1.2, p. 88, in Vrabie [25], we conclude that $f(s) \in F(s, u(s))$ for each $k \geq 1$ and a.e. for $s \in[\tau, T] \backslash \mathcal{O}_{k}$. Since $\lim _{k} \lambda\left(\mathcal{O}_{k}\right)=0$, we deduce that

$$
f(s) \in F(s, u(s))
$$

a.e. for $s \in[\tau, T]$.

Finally, taking into account that $A$ is of compact type — see Definition 8 - and passing to the limit both sides in (7), for $n \rightarrow \infty$, we get

$$
u(t)=u(t, \tau, \xi, f),
$$

for each $t \in[\tau, T]$. Since, by (i), (iii), (vi) and (vii) in Lemma 1, we have $u_{n}\left(a_{n}(t)\right) \in K\left(a_{n}(t)\right.$ ), $u_{n}(T) \in K(T), a_{n}(t) \uparrow t$, as $n \rightarrow \infty$, uniformly for $t \in[\tau, T), \lim _{n} u_{n}\left(a_{n}(t)\right)=\lim _{n} u_{n}(t)=u(t)$ uniformly for $t \in[\tau, T]$, and $\mathcal{K}$ is locally closed from the left, it follows that $u(t) \in K(t)$ for each $t \in[\tau, T]$. By (8), we conclude that $u$ is a $C^{0}$ - solution of (1), and this completes the proof.

\section{A comparison result}

Let $H$ be a real Hilbert space with inner product $\langle\cdot, \cdot\rangle$, let $C \subseteq H$ be a closed convex cone with $C \cap(-C)=\{0\}$, let “ $\preceq$ ” be the partial order on $H$ defined by $C$, i.e., $x \preceq y$ if and only if $y-x \in C$. Let $\varphi: H \rightarrow \mathbb{R}_{+} \cup\{+\infty\}$ be a proper, 1.s.c., convex function and let $\partial \varphi: D(\partial \varphi) \subseteq H \rightsquigarrow H$ be the subdifferential of $\varphi$. It is known that $-\partial \varphi$ is the infinitesimal generator of a nonlinear semigroup of contractions $\{S(t): \overline{D(\partial \varphi)} \rightarrow \overline{D(\partial \varphi)} ; t \geq 0\}$. Let $a: I \rightarrow D(\partial \varphi)$ be a continuous function and let $K: I \rightsquigarrow H$ be defined by $K(t):=\{x \in H ; a(t) \preceq x\}$ for each $t \in I$. Let $\mathcal{K}$ be the graph of $K$ and $F: \mathcal{K} \rightsquigarrow H$ be a given multi-function. We are interested in finding sufficient conditions in order that $\mathcal{K}$ be strongly-viable with respect to $-\partial \varphi+F$, i.e., in order that, for each $(\tau, \xi) \in I \times H$ with $a(\tau) \preceq \xi$, to exists at least one strong-solution $u$, on $[\tau, T]$, of the problem

$$
\left\{\begin{array}{l}
u^{\prime}(t) \in-\partial \varphi(u(t))+F(t, u(t)) \\
u(\tau)=\xi \\
a(t) \preceq u(t) \quad \text { for each } t \in[\tau, T],
\end{array}\right.
$$

i.e. a continuous function $u:[\tau, T] \rightarrow D(\partial \varphi)$ with $u \in W^{1,2}(\tau, T ; H)$ and for which there exists $f \in L^{2}(\tau, T ; H)$ such that:

$\left(S_{1}\right) \quad u^{\prime}(t) \in-\partial \varphi(u(t))+f(t), \quad$ a.e. for $t \in[\tau, T]$;

$\left(S_{2}\right) \quad f(t) \in F(t, u(t)), \quad$ a.e. for $t \in[\tau, T]$;

$\left(S_{3}\right) \quad u(\tau)=\xi$

$\left(S_{4}\right) \quad a(t) \preceq u(t), \quad$ for each $t \in[\tau, T]$.

For a thorough study of problems of this kind, with $F$ single-valued and independent of $u$, that is $F(t, u)=\{f(t)\}$, and without the monotonicity constraint $\left(S_{4}\right)$, see Brezis [6]. 
Definition 11 We say that a convex function $\varphi: H \rightarrow \mathbb{R}_{+} \cup\{+\infty\}$ is of compact type if for each $k>0$, the level set

$$
\mathcal{L}_{k}=\left\{u \in H ;\|u\|^{2}+\varphi(u) \leq k\right\}
$$

is relatively compact in the norm topology of $H$.

Remark 5 If $\varphi: H \rightarrow \mathbb{R}_{+} \cup\{+\infty\}$ is a proper, l.s.c., convex function of compact type and $\partial \varphi$ is its subdifferential, then $A=-\partial \varphi$ generates a compact semigroup — see Vrabie [25, Proposition 2.2.2, p. 42], and is of compact type in the sense of Definition 8 -Vrabie [25, Corollary 2.3.2, p. 50].

Theorem 7 Let $\varphi: H \rightarrow \mathbb{R}_{+} \cup\{+\infty\}$ be a proper, l.s.c., convex function of compact type with $\partial \varphi$ singlevalued, let $a \in W_{\mathrm{loc}}^{1,1}(I ; H)$, with $a(t) \in D(\partial \varphi)$ for each $t \in I$, let $C \subseteq \overline{D(\partial \varphi)}$ be a closed convex cone with $C \cap(-C)=\{0\}$ and $\overline{D(\partial \varphi) \cap C}=C$, and let $\mathcal{K}$ be the graph of the multi-function $K: I \rightsquigarrow H$ defined by $K(t)=a(t)+C$ for $t \in I$. Let us assume that $S(t) C \subseteq C$ for each $t \geq 0$, and $\mathcal{K}$ is $-\partial \varphi-C^{0}$-viable by itself. Let us further assume that $F$ is a nonempty, convex and weakly compact valued multi-function which is essentially locally bounded and almost strongly-weakly u.s.c. Then, a sufficient condition in order that $\mathcal{K}$ be $C^{0}$-viable with respect to $-\partial \varphi+F$ is to exists a negligible set $N \subseteq I$ such that, for each $\tau \in I \backslash N$ and each $\xi \in \partial C \cap D(\partial \varphi)$, we have

$$
\operatorname{dist}\left(-\partial \varphi(a(\tau)+\xi)+\partial \varphi(\xi)-a^{\prime}(\tau)+F(\tau, a(\tau)+\xi) ; C\right)=0
$$

Proof. Throughout, $O, O_{1}, \ldots, O_{4}$, denote some functions defined on $(0,1)$ with values in $H$, with $\lim _{h \downarrow 0} O(h)=\lim _{h \downarrow 0} O_{1}(h)=\cdots=\lim _{h \downarrow 0} O_{4}(h)=0$.

First, let us notice that, for every $h \in(0,1), \xi \in D(\partial \varphi)$ and $\eta \in H$, we have

$$
\left\{\begin{array}{l}
a(\tau+h)=a(\tau)+h a^{\prime}(\tau)+h O(h) \\
u(\tau+h, \tau, \xi, \eta)=\xi-h \partial \varphi(\xi)+h \eta+h O(h) \\
S(h) \xi=\xi-h \partial \varphi(\xi)+h O(h) .
\end{array}\right.
$$

To prove that (9) implies the tangency condition

$$
F(\tau, a(\tau)+\xi) \in \mathcal{T S}_{\mathcal{K}}^{-\partial \varphi}(\tau, a(\tau)+\xi),
$$

for each $\tau \in I \backslash N$ and each $\xi \in C \cap D(\partial \varphi)$, let us observe that, in view of (10), for each $\eta \in F(\tau, a(\tau)+\xi)$, we have

$$
\begin{aligned}
\operatorname{dist}( & u(\tau+h, \tau, a(\tau)+\xi, \eta) ; K(\tau+h)) \\
& =\operatorname{dist}(u(\tau+h, \tau, a(\tau)+\xi, \eta) ; a(\tau+h)+C) \\
& =\operatorname{dist}\left(a(\tau)+\xi+h[-\partial \varphi(a(\tau)+\xi)+\eta]+h O_{1}(h) ; a(\tau)+h a^{\prime}(\tau)+h O_{2}(h)+C\right) \\
& =\operatorname{dist}\left(\xi-S(h) \xi+h\left[-\partial \varphi(a(\tau)+\xi)-a^{\prime}(\tau)+\eta\right]+h O_{3}(h) ;-S(h) \xi+C\right) \\
& =\operatorname{dist}\left(h\left[-\partial \varphi(a(\tau)+\xi)+\partial \varphi(\xi)-a^{\prime}(\tau)+\eta\right]+h O_{4}(h) ;-S(h) \xi+C\right) .
\end{aligned}
$$

Since, for each $\xi \in C \cap D(\partial \varphi)$ and each $h>0$, we have $S(h) C \subseteq C$ and $C$ is a convex cone, it follows that

$$
C \subseteq-S(h) \xi+C \text { and } h C=C .
$$

Let now $\eta \in F(\tau, a(\tau)+\xi)$ be arbitrary but fixed. From (10), (12) and (13), we get

$$
\begin{aligned}
\operatorname{dist} & (u(\tau+h, \tau, a(\tau)+\xi, F(\tau, a(\tau)+\xi)) ; K(\tau+h)) \\
& \leq \operatorname{dist}(u(\tau+h, \tau, a(\tau)+\xi, \eta) ; K(\tau+h)) \\
& \leq \operatorname{dist}\left(h\left[-\partial \varphi(a(\tau)+\xi)+\partial \varphi(\xi)-a^{\prime}(\tau)+\eta+O_{4}(h)\right] ; C\right)
\end{aligned}
$$




$$
\begin{aligned}
& =\operatorname{dist}\left(h\left[-\partial \varphi(a(\tau)+\xi)+\partial \varphi(\xi)-a^{\prime}(\tau)+\eta+O_{4}(h)\right] ; h C\right) \\
& =h \operatorname{dist}\left(-\partial \varphi(a(\tau)+\xi)+\partial \varphi(\xi)-a^{\prime}(\tau)+\eta+O_{4}(h) ; C\right) \\
& \leq h \operatorname{dist}\left(-\partial \varphi(a(\tau)+\xi)+\partial \varphi(\xi)-a^{\prime}(\tau)+\eta ; C\right)+h\left\|O_{4}(h)\right\| \\
& =h\left\|O_{4}(h)\right\| .
\end{aligned}
$$

Dividing by $h$ and passing to the limit for $h \downarrow 0$, we deduce

$$
\begin{gathered}
\liminf _{h \downarrow 0} \frac{1}{h} \operatorname{dist}(u(\tau+h, \tau, a(\tau)+\xi, F(\tau, a(\tau)+\xi)) ; K(\tau+h)) \\
\leq \operatorname{dist}\left(-\partial \varphi(a(\tau)+\xi)+\partial \varphi(\xi)-a^{\prime}(\tau)+\eta ; C\right)
\end{gathered}
$$

for each $\eta \in F(\tau, a(\tau)+\xi)$. Since for each $\xi \in \partial C \cap D(\partial \varphi)$, we have

$$
\begin{aligned}
\inf _{\eta \in F(\tau, a(\tau)+\xi)} & \operatorname{dist}\left(-\partial \varphi(a(\tau)+\xi)+\partial \varphi(\xi)-a^{\prime}(\tau)+\eta ; C\right) \\
= & \operatorname{dist}\left(-\partial \varphi(a(\tau)+\xi)+\partial \varphi(\xi)-a^{\prime}(\tau)+F(\tau, a(\tau)+\xi) ; C\right)
\end{aligned}
$$

and, by (9), the latter equals 0 , we conclude that (11) holds true. If $\xi \in(C \backslash \partial C) \cap D(\partial \varphi)$, the conclusion above follows from the simple remark that, for $h>0$ small enough,

$$
\operatorname{dist}(u(\tau+h, \tau, a(\tau)+\xi, \eta) ; K(\tau+h))=\operatorname{dist}(u(\tau+h, \tau, a(\tau)+\xi, \eta) ; a(\tau+h)+C)=0 .
$$

So (11) holds true for each $\xi \in C \cap D(\partial \varphi)$, and thus we are in the hypotheses of Theorem 5 -see also Remark 5. This completes the proof.

Remark 6 Since $F(\tau, a(\tau)+\xi)$ is convex and weakly compact and $C$ is convex and closed, the condition (9) is equivalent to: for each $\tau \in I \backslash N$ and each $\xi \in \partial C \cap D(\partial \varphi)$, there exists $\eta \in F(\tau, a(\tau)+\xi)$ such that

$$
-\partial \varphi(a(\tau)+\xi)+\partial \varphi(\xi)-a^{\prime}(\tau)+\eta \in C .
$$

Remark 7 In the semi-linear case, i.e. $\partial \varphi=A$ with A linear, we have a sufficient condition better than (9). Namely, if $\partial \varphi$ is linear, in order that $\mathcal{K}$ be $C^{0}$-viable with respect to $-\partial \varphi+F$ it suffices to exists a negligible set $N \subseteq I$ such that, for each $\tau \in I \backslash N$ and each $\xi \in \partial C$

$$
A a(\tau)-a^{\prime}(\tau)+F(\tau, a(\tau)+\xi) \in \mathcal{T S}_{C}^{A}(\xi) .
$$

For details, see Necula, Popescu, Vrabie [19].

Acknowledgement. We acknowledge the support of the PN-II-ID-PCE-2007-1, Grant ID_ 397, (for the first two authors), as well as the support of "O. Mayer" Institute of Mathematics and of Marie-CurieITN "Controlled Systems", no. 213841/2008 (for the third author).

\section{References}

[1] Bebernes, W. And Schuur, I. D., (1970). The Ważewski topological method for contingent equations, Ann. Mat. Pura Appl., 87, 271-278.

[2] Benilan, Ph., (1972). Equations d'évolution dans un espace de Banach quelconque et applications, Thèse, Orsay.

[3] Bothe, D., (1992). Multivalued differential equations on graphs, Nonlinear Anal., 18, 3, 245-252. DOI: 10.1016 /0362-546X(92)90062-J 
[4] Bothe, D., (1992). Multivalued differential equations on graphs and applications, Ph. D. Thesis, Paderborn.

[5] Bouligand, H., (1930). Sur les surfaces dépourvues de points hyperlimités, Ann. Soc. Polon. Math., 9, 32-41.

[6] BreZIS, H., (1972). Problèmes unilatéraux, J. Math. Pures Appl., 51, 1-168.

[7] BREZIS, H. AND BRowder, F. E., (1976). A general principle on ordered sets in nonlinear functional analysis, Adv. in Mathematics, 21, 3, 355-364. DOI: 10.1016/S0001-8708(76)80004-7

[8] CÂrJă, O., Necula, M. And Vrabie, I. I., (2008). Necessary and sufficient conditions for viability for nonlinear evolution inclusions, Set-Valued Analysis, 16, 5-6, 701-731. DOI: 10.1007/s11228-007-0063-7

[9] CÂrJă, O., Necula, M. And Vrabie, I. I., (2007). Viability, Invariance and Applications, North-Holland Mathematics Studies, 207, Elsevier.

[10] CÂRJĂ, O., Necula, M. AND VRabie, I. I., (2009). Necessary and sufficient conditions for viability for semilinear differential inclusions, Trans. Amer. Math. Soc., 361, 1, 343-390. DOI: 10.1090/S0002-9947-08-04668-0

[11] CÂrJă, O., Necula, M. And Vrabie, I. I., (2009). Tangent Sets, Viability for Differential Inclusions and Applications, Nonlin. Anal., 71, e979-e990. DOI: 10.1016/j.na.2009.01.055

[12] Crandall, M. G. And Liggett, T. M., (1971). Generation of semi-groups of nonlinear transformations in general Banach spaces, Amer. J. Math., 93, 2, 265-298. DOI: 10.2307/2373376

[13] DiaZ, J. I. And VRabie, I. I., (1994). Compactness of the Green Operator of nonlinear diffusion equations: application to Boussinesq type systems in fluid mechanics, Topol. Methods in Nonlinear Anal., 4, 399-416.

[14] Lakshmikantham, V. And Leela, S., (1981). Nonlinear differential equations in abstract spaces, International Series in Nonlinear Mathematics, 2, Pergamon Press.

[15] Methlouthi, H., (1977). Équations différentielles multivoques sur une graphe locallement compact, $C$. $R$. Acad. Sc. Paris, Série A, 284, 1287-1290.

[16] Nagumo, M., (1942). Über die Lage der Integralkurven gewöhnlicher Differentialgleichungen, Proc. Phys.Math. Soc. Japan, 24, 551-559.

[17] Necula, M., (2002). Viability of variable domains for differential equations governed by Carathéodory perturbations of nonlinear $m$-accretive operators, An. Ştiinţ. Univ. Al. I. Cuza Iaşi Secţ. I a Mat., 48, 41-60.

[18] Necula, M., Popescu, M. And Vrabie, I. I., (2008). Viability for differential inclusions on graphs, SetValued Analysis, 16, 7-8, 961-981. DOI: 10.1007/s11228-008-0090-z

[19] Necula, M., Popescu, M. And Vrabie, I. I., (2009). Evolution equations on locally closed graphs and applications, Nonlin. Anal., 71, e2205-e2216. DOI: 10.1016/j.na.2009.04.044

[20] Pavel, N. H., (1977). Invariant sets for a class of semi-linear equations of evolution, Nonlinear Anal., 1, 2 , 187-196. DOI: 10.1016/0362-546X(77)90009-8

[21] Pavel, N. H. And Vrabie, I. I., (1978). Équations d'évolution multivoques dans des espaces de Banach, C. R. Acad. Sci. Paris, Sér. I Math., 287, 315-317.

[22] Perron, O., (1915). Ein neuer Existenzbeweis für Integrale der Differentialgleichung, $y^{\prime}=f(x, y)$, Math. Ann., 76, 471-484.

[23] Severi, F., (1931). Su alcune questioni di topologia infinitesimale, Annales Soc. Polonaise, 9, 97-108. 
[24] VRABIE, I. I., (1981). Compactness methods and flow-invariance for perturbed nonlinear semigroups, An. Ştiints. Univ. “Al. I. Cuza” Iaşi Secţ. I a Mat., 27, 117-125.

[25] VRABIE, I. I., (1995). Compactness methods for nonlinear evolutions, Second Edition, Pitman Monographs and Surveys in Pure and Applied Mathematics, 75, Longman.

[26] VRabie, I. I., (2003). C C-semigroups and applications, North-Holland Publishing Co., Amsterdam.

\section{Mihai Necula}

Faculty of Mathematics,

"Al. I. Cuza" University, Iaşi 700506,

Romania

necula@uaic.ro

\section{Marius Popescu}

Faculty of Sciences,

University "Dunărea de Jos",

Galaţi 800201,

Romania

Marius.Popescu@ugal.ro

\section{Ioan I. Vrabie}

"O. Mayer" Institute of Mathematics,

Romanian Academy,

Iaşi 700506

Romania

ivrabie@uaic.ro 